\title{
P10 Oscillatory Flow Patterns in Peripheral Conduit Artery are Related to Central Arterial Stiffness in Healthy Subjects
}

\author{
Smriti Badhwar*, Dinu Chandran, Ashok Jaryal, Rajiv Narang, Chetan Patel, Kishore Kumar Deepak \\ All India Institute of Medical Sciences, New Delhi, India
}

\section{ABSTRACT}

Background: Studies in endothelial cell cultures show that oscillating flow pattern leads to endothelial dysfunction [1]. The only factor known to contribute towards oscillatory flows is peripheral arterial conductance [2]. However, contribution of alterations in central arterial properties to oscillatory flows is not established.

Methods: Healthy subjects $(n=28)$ (Age $51.0 \pm 9.4$ years) with resting retrograde flow in brachial artery were recruited for the study. Pulsed-wave doppler (M7, MindRay) was used for estimation of blood flow velocity with simultaneous diameter acquisition for shear rate calculation. Oscillatory Shear Index (OSI) was calculated by: Retrograde shear rate/(Anterograde shear rate + Retrograde shear rate). Local and regional central arterial stiffness was estimated using carotid distensibility, compliance and Incremental Elastic Modulus (IEM) (Medical Imaging Applications) and carotid-femoral Pulse Wave Velocity (PWV) using applanation tonometry (SphygmocorÒ) respectively. Peripheral vascular stiffness was assessed by carotid-radial PWV. Forearm vascular resistance was estimated using peripheral mean arterial pressure and mean anterograde flow. Carotid-Intima-MediaThickness (CIMT) was measured using B-mode ultrasound for subclinical atherosclerotic burden estimation.

Results: Retrograde flow showed a significant positive correlation with IEM, CIMT and Augmentation Index (AI) $(r=0.64$, 0.47 and 0.62 respectively) (Figures 1 and 2). A significant negative correlation was seen between retrograde flow and carotid compliance $(r=-0.58)$. OSI correlated positively with IEM $(r=0.56)$ and AI $(r=0.45)$.

Conclusion: Results of the current study suggest that oscillatory flow patterns in peripheral conduit artery could originate due to decrease in central arterial compliance possibly because of its impact on the incident pressure wave.

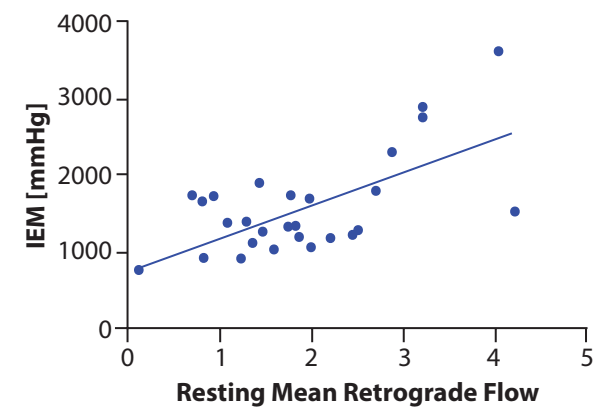

Figure 1 Correlation between brachial artery mean retrograde flow at rest and carotid artery Incremental Elastic Modulus.

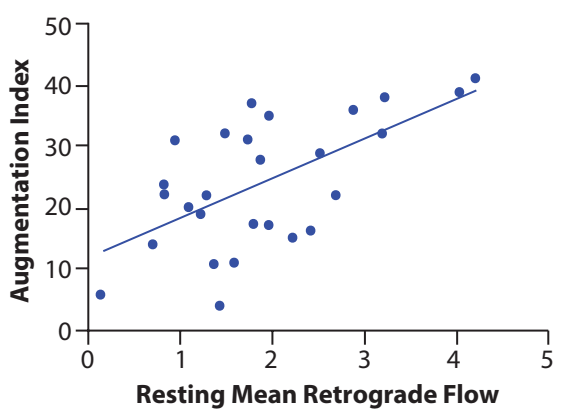

Figure 2 Correlation between brachial artery mean retrograde flow at rest and aortic Augmentation Index. 


\section{REFERENCES}

[1] Jenkins NT, Padilla J, Boyle LJ, Credeur DP, Laughlin MH, Fadel PJ. Disturbed blood flow acutely induces activation and apoptosis of the human vascular endothelium. Hypertension 2013;61:615-21.

[2] Padilla J, Young CN, Simmons GH, Deo SH, Newcomer SC, Sullivan JP, et al. Increased muscle sympathetic nerve activity acutely alters conduit artery shear rate patterns. Am J Physiol Heart Circ Physiol 2010;298:H1128-H35.

(C) 2019 Association for Research into Arterial Structure and Physiology. Publishing services by Atlantis Press International B.V. This is an open access article distributed under the CC BY-NC 4.0 license (http://creativecommons.org/licenses/by-nc/4.0/). 